\title{
Quasi-geostrophic dynamics in the presence of moisture gradients
}

\author{
Joy M. Monteiro and Jai Sukhatme \\ Centre for Atmospheric and Oceanic Sciences, \\ Indian Institute of Science, \\ Bangalore 560012, India
}

\begin{abstract}
The derivation of a quasi-geostrophic (QG) system from the rotating shallow water equations on a midlatitude $\beta$-plane coupled with moisture is presented. Condensation is prescribed to occur whenever the moisture at a point exceeds a prescribed saturation value. It is seen that a slow condensation time scale is required to obtain a consistent set of equations at leading order. Further, since the advecting wind fields are geostrophic, changes in moisture (and hence, precipitation) occur only via non-divergent mechanisms. Following observations, a saturation profile with gradients in the zonal and meridional directions is prescribed. A purely meridional gradient has the effect of slowing down the dry Rossby waves, through a reduction in the "equivalent gradient" of the background potential vorticity. A large scale unstable moist mode results on the inclusion of a zonal gradient by itself, or in conjunction with a meridional moisture gradient. For gradients that are are representative of the atmosphere, the most unstable moist mode propagates zonally in the direction of increasing moisture, matures over an intraseasonal timescale and has small phase speed.
\end{abstract}

Journal Ref: QJRMS, DOI:10.1002/qj.2644, 2015.

\section{INTRODUCTION}

Following [1], the study of idealised dynamical models forced by active, condensable scalars has received considerable attention, mainly due to the fact that these models represent the action of water vapour on the atmosphere in a simple manner. From a tropical perspective, the use of one or two mode shallow water systems on an equatorial $\beta$-plane is prevalent, and a large body of work had been directed towards understanding the so-called convectively coupled equatorial waves, i.e., to identify the effects of moisture on "dry" equatorial waves [see for example, the recent review by 2]. The vertical decomposition into modes has been done in many ways: for example, using analytic functions that satisfy appropriate boundary conditions [3], basis functions derived from observations [4]. Another avenue has been to try and see if the addition of moisture gives rise to modes that do not exist in the traditional "dry" equations [see, for example, $5,6]$.

Outside the tropics, the influence of condensational heating via a moisture equation on the linear evolution of a quasi-geostrophic (QG) system [7], barotropic [8] and baroclinic instability [9, 10], the life-cycle of baroclinic eddies [11] and homogeneous QG turbulence [12] has also been considered. More pertinent to our work has been the study of "diabatic Rossby waves" which are generated purely due to PV anomalies rsulting from latent heat release [13-15]. Interestingly, applications to other planetary atmospheres, such as Mars with $\mathrm{CO}_{2}$ condensation and Titan with $\mathrm{CH}_{4}$ condensation, have also been successfully pursued $[16,17]$.

In the context of QG dynamics, forcing due to condensation is usually directly appended to the "dry" system which makes it difficult to understand the constraints (if any) that the forcing must obey to be consistent with the requirements of QG theory. Furthermore, most previous studies prescribe a homogeneous background saturation profile which does not include horizontal gradients. A noteworthy exception is [5]; by allowing a meridional gradient in the saturation profile, their linear analysis on an $f$-plane showed the emergence of a remarkable unstable eastward propagating mode that owed its existence to the aforementioned meridional gradient. As meridional and zonal gradients in precipitable water are robust features of the atmosphere both in the mid-latitudes (Figure 1) and the tropics [18], it is natural to incorporate them along with a gradient in the planetary vorticity when formulating simple models to study the effects of moisture on large scale dynamics. In fact, following [5], a numerical calculation with zonal and meridional moisture gradients was carried out on the equatorial $\beta$-plane by [19]. However, the complexity of the full shallow water system did not permit a complete understanding of the mechanism behind the observed instability.

In this paper, a QG system is derived from the moist shallow water equations (SWE) on a midlatitude $\beta$-plane. This clearly delineates the constraints that the moist forcing must satisfy in order to maintain geostrophy at leading order. We then proceed to a linear analysis in the presence of both meridional and zonal gradients of the saturation profile. With only a meridional gradient, there are no unstable modes and the Rossby waves that emerge are slower than their dry counterparts. Interestingly, the slowing down of the waves is traced to a reduction in the "equivalent gradient" of the background potential vorticity. It is seen that in the presence of zonal gradients, the system supports a large scale unstable moist mode. In fact, with both meridional and zonal gradients in the saturation profile, with values similar to those observed in the atmosphere, the most unstable mode propagates zonally in a direction of increasing moisture, matures over an intraseasonal timescale and is characterised by a small 

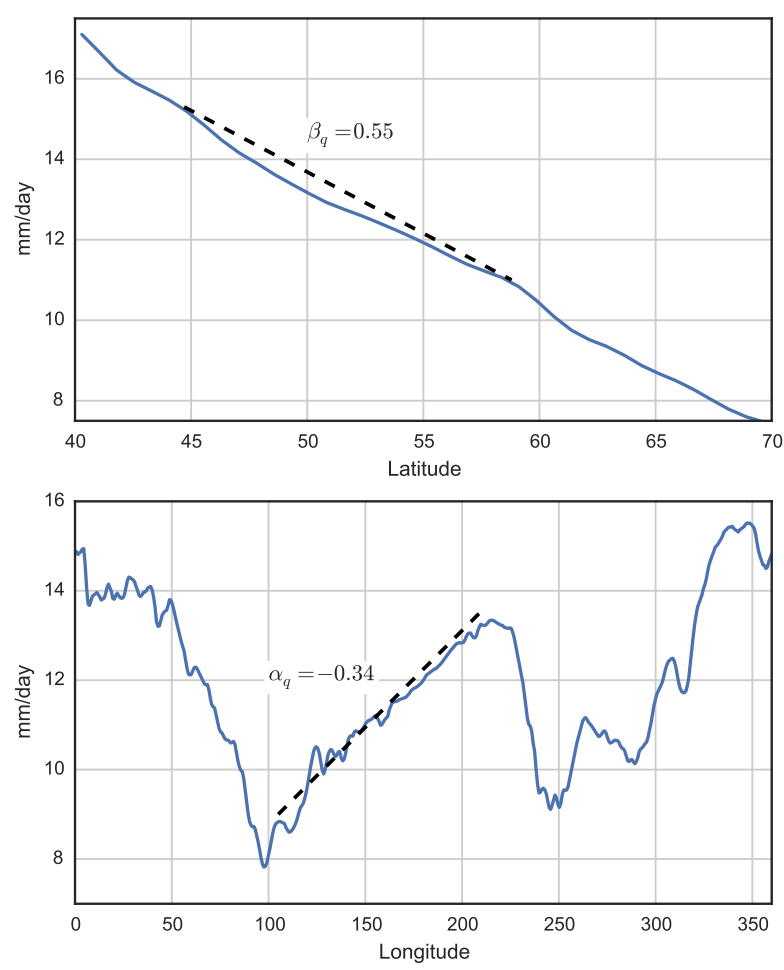

FIG. 1: Panel (a) shows the meridional profile of the zonally averaged climatological precipitable water in the ERAInterim dataset between $40-70 \mathrm{~N}$. This corresponds to a nondimensional $\beta_{q}$ (see Section 2.3) of about 0.55. Panel (b) shows the zonal profile of precipitable water averaged between 40-70N. A strong gradient is seen in both the Pacific and Atlantic oceans, corresponding to a non-dimensional $\alpha_{q}$ of about -0.34 (see Section 2.3).

phase speed. The eigenvector structure corresponding to the waves in the presence of gradients is also examined to determine the phase relationship between moistening and precipitation. Finally, a summary of results and a brief discussion conclude the paper.

\section{DERIVATION OF THE MODEL}

In deriving our model, we follow [20] and consider the one-layer forced SWE. In this situation, the forcing due to condensation manifests itself as a mass source/sink [21], and the equations read

$$
\begin{gathered}
\frac{D u}{D t}-f v=-g h_{x}, \\
\frac{D v}{D t}+f u=-g h_{y}, \\
\frac{D h}{D t}+h(\nabla \cdot \mathbf{u})=-\chi S, \\
\frac{D q}{D t}+q(\nabla \cdot \mathbf{u})=-S .
\end{gathered}
$$

Here $u, v$ are the horizontal velocities, $h$ is the height of the shallow water system, $f=f_{0}+\beta y$ is the Coriolis parameter and $q$ is the column water vapour. $S=$ $\delta\left(q-q_{s}\right) / \tau_{q}$ is a Betts-Miller like condensation sink for the water vapour equation, where $\tau_{q}$ is the condensation timescale and $\delta$ is one when $q>q_{s}$ and zero otherwise. $q_{s}$ is the (prescribed) background saturation water vapour profile. With a "conversion factor" $\chi$ this takes the form of a mass source/sink. We note that this particular parameterisation of condensation is reminiscent of the model developed by [22], where precipitation is a function of column water vapour rather than the saturation vapour pressure [see also the discussion in 23]. Writing $q=q_{s}+\tilde{q}$, the source term reduces to $S=\delta \tilde{q} / \tau_{q}$, and the vapour conservation law takes the form:

$$
\frac{D \tilde{q}}{D t}+\tilde{q}(\nabla \cdot \mathbf{u})+\nabla \cdot\left(\mathbf{u} q_{s}\right)=-\frac{\delta}{\tau_{q}} \tilde{q}
$$

where $q_{s}=q_{s}(x, y)$ is a function of space only, whereas $\tilde{q}=\tilde{q}(x, y, t)$ is also a function of time.

\section{A. Conservation Laws}

The "dry" shallow water equations respect the material invariance of potential vorticity $(f+\zeta) / h$, as well as the global conservation of energy $E=\left(g h^{2}+h \mathbf{u} \cdot \mathbf{u}\right) / 2$. Eliminating $S$ from the last two equations in Eq. 1 to give a single conservation law, the equivalent statements for (1) are:

$$
\begin{array}{r}
\frac{D}{D t}\left(\frac{f+\zeta}{m}\right)=0, \\
\frac{d}{d t} \int_{\Omega} E_{m}=\chi \int_{\Omega} q \nabla \cdot(h \mathbf{u})-q^{2}(\nabla \cdot \mathbf{u}) / 2,
\end{array}
$$

where $\Omega$ is any area over which the divergence is assumed zero. $E_{m}=\left(g m^{2}+m \mathbf{u} . \mathbf{u}\right) / 2$, where $m=h-\chi q$, a simplified representation of the moist static energy.

\section{B. Asymptotic Analysis}

On a $\beta$-plane, we scale the equations as: $u, v \sim$ $U(u, v), t \sim L / U, h \sim H+P \eta$ and $\tilde{q}, q_{s} \sim Q_{0}$. Non-dimensionalising using the shallow water definitions of Rossby Number $\left(R o=U / f_{0} L\right)$, Froude Number $(F r=U / \sqrt{g H})$, relative height of waves $(\Gamma=P / H)$, ratio of condensation time scale to advective time scale $\left(\alpha=\tau_{q} /(L / U)\right)$ and strength of the $\beta$-effect $\left(r=\beta L / f_{0}\right)$, 
(1) takes the form

$$
\begin{array}{r}
\frac{D u}{D t}-\frac{1}{R o}(v+r v y)=-\frac{\Gamma}{F r^{2}} \eta_{x}, \\
\frac{D v}{D t}+\frac{1}{R o}(u+r u y)=-\frac{\Gamma}{F r^{2}} \eta_{y}, \\
\frac{D \eta}{D t}+\frac{1}{\Gamma}(\nabla \cdot \mathbf{u})+\eta(\nabla \cdot \mathbf{u})=-\delta \frac{\chi Q_{0} \tilde{q}}{P \alpha}, \\
\frac{D \tilde{q}}{D t}+\tilde{q}(\nabla \cdot \mathbf{u})+\nabla \cdot\left(\mathbf{u} q_{s}\right)=-\frac{\delta}{\alpha} \tilde{q},
\end{array}
$$

where $Q_{0}$ is the scale parameter for $\tilde{q}$. Taking the limit of small Rossby, Froude and perturbations $(R o=F r=$ $\Gamma=r=\epsilon)$, using a "slow" mass source $(\alpha=1)$ and assuming an $\mathcal{O}(1)$ contribution to the height field from $\chi Q_{0} / P$, we get

$$
\begin{aligned}
\frac{D u}{D t}-\frac{1}{\epsilon}(v+\epsilon v y) & =-\frac{1}{\epsilon} \eta_{x}, \\
\frac{D v}{D t}+\frac{1}{\epsilon}(u+\epsilon u y) & =-\frac{1}{\epsilon} \eta_{y}, \\
\frac{D \eta}{D t}+\frac{1}{\epsilon}(\nabla \cdot \mathbf{u})+\eta(\nabla \cdot \mathbf{u}) & =-\delta \tilde{q}, \\
\frac{D \tilde{q}}{D t}+\tilde{q}(\nabla \cdot \mathbf{u})+\nabla \cdot\left(\mathbf{u} q_{s}\right) & =-\delta \tilde{q} .
\end{aligned}
$$

Expanding all fields in an asymptotic series in powers of $\epsilon$, and collecting the dominant terms $(\mathcal{O}(1 / \epsilon))$, we obtain the following balance,

$$
v^{0}=\eta_{x}^{0}, u^{0}=-\eta_{y}^{0}, \nabla \cdot \mathbf{u}^{\mathbf{0}}=0 .
$$

Which is nothing but a non-divergent geostrophic flow. Note that, with $\chi Q_{0} / N \sim \mathcal{O}(1)$, if we assume "fast" condensation and use $\alpha=\epsilon$, we end up with an inconsistent set of equations at $\mathcal{O}(1 / \epsilon)$. Thus, a "slow" mass source is essential to maintain geostrophy in the leading order fields. Also, this time scale is independent of the functional form of the forcing, which need not be closed on the vapour equation as has been done in our formulation.

Proceeding with the asymptotic expansion, at $\mathcal{O}(1)$, we have,

$$
\begin{gathered}
\frac{D^{0} u^{0}}{D t}-v^{1}-v^{0} y=-\eta_{x}^{1}, \\
\frac{D^{0} v^{0}}{D t}+u^{1}+u^{0} y=-\eta_{y}^{1}, \\
\frac{D^{0} \eta^{0}}{D t}+\left(\nabla \cdot \mathbf{u}^{\mathbf{1}}\right)=-\delta \tilde{q}^{0}, \\
\frac{D^{0} \tilde{q}^{0}}{D t}+\nabla \cdot\left(\mathbf{u}^{0} q_{s}\right)=-\delta \tilde{q}^{0} .
\end{gathered}
$$

Taking the curl of the first two equations and substituting for $\nabla \cdot \mathbf{u}^{\mathbf{1}}$ from the third, we get (dropping superscripts as all fields are at order zero) a "QG-like" vorticity equation coupled with moisture advection by the order zero velocity fields. Specifically,

$$
\begin{aligned}
\frac{D}{D t}\left(\nabla^{2} \eta-\eta\right)+\eta_{x}-\delta \tilde{q} & =0 \\
\frac{D \tilde{q}}{D t}+(\mathbf{u} \cdot \nabla) q_{s}+\delta \tilde{q} & =0
\end{aligned}
$$

Equations (9) represents the "slow" evolution of the moist rotating shallow water system (1). We emphasise that, in (9), the time scale at which the effects of condensation manifest themselves is of the same order as the advective time scale.

Understandably, most applications of a moist framework have been in tropical settings, and in contrast to the present scenario, the time scale of condensation is usually taken to be much smaller than that of advection. In fact, a limit that has proved useful in tropical analysis is $\tau_{q} \rightarrow 0$, the so-called limit of rapid condensation [see for example, 24, 25, for dynamic and kinematic applications, respectively] or strict quasi-equilibrium (SQE) [26]. Midlatitude studies which look at the effect of latent heat release on baroclinic waves generally use different moisture parameterisations: in particular, wave-CISK and largescale rain schemes [27]. Wave-CISK closes the heating on the QG vertical velocity $(w)$ at some level (for example, the cloud base $z_{b}$ ) [i.e., $Q \propto w\left(z_{B}\right) h(z)$ ] where $h(z)$ is the vertical profile of heating. Large scale rain schemes close the heating via $Q \propto w(z) r(z)$, where $r(z)$ is the vertical specific humidity profile. In both these cases, the vertical profile of heating/humidity is important. Further, these schemes usually do not consider the effects of horizontal gradients of moisture.

In the tropical context, the idea of using forcing due to condensation as a slow, second order term in an asymptotic expansion has been explored by [28]. Furthermore, in the lifecycle of mesoscale convective systems, [29] observe significant moisture anomalies in the free troposphere for as much as four days before and after peak rainfall, and suggest that the long duration of these anomalies might project mesoscale variability onto intraseasonal scales. In a different context, [30] showed that moist convective response time must exceed the lifetime of a single cumulus cloud to account for the observed phase of the semidiurnal tide. More broadly, "slow" forcing of the full SWE, that projects mainly on the balanced dynamics, has been adopted in models that study the effect of moist convection on large scale circulation in the atmospheres of Jupiter and Saturn [[31], see also the discussion in [32]]. In addition, for slow, large scale modes such as the Madden-Julian Oscillation (MJO), studies have shown the importance of the horizontal advection of moist static energy [see, for example, 33-36]. Also, convective parameterisations that are based on relative humidity are seen to perform better than those based on convergence in simulating the MJO [37]. In fact, a "mechanism-denial" experiment showed that the MJO shuts off in a GCM when the rotational component of horizontal moist static energy advection is removed [35]. Thus, given the slow time scales of the rotational modes in the atmosphere, we argue that the main features of our moist model, namely a) slow time scales, b) nondivergent advection and c) relative (column) humidity based latent heat release can be justified on physical grounds. 


\section{Vapour Equation}

To facilitate study on a doubly periodic domain, we follow previous work and assume a linear gradient in the meridional direction of the saturation vapour specific humidity [5]. In addition, based on Figure 1, we also consider a linear dependence of $q_{s}$ with longitude. Even though the temperature may be fairly uniform in longitude, which leads to a uniform $q_{s}$ from the ClausiusClapeyron relation, it should be remembered that this relation is an upper bound. Indeed, the general circulation of the atmosphere gives character to the $q_{s}$ profile.

Therefore, a simple prescription for the saturation profile reads, $q_{s}=Q_{0}\left(1-\beta_{q} \frac{y}{L}-\alpha_{q} \frac{x}{L}\right)$. As $\alpha_{q}$ can be positive or negative, we have $\alpha_{q}, \beta_{q}<1$ individually, and $\alpha_{q}+\beta_{q}<1$ in combination to enforce positivedefiniteness of $q_{s}$. Due to the scaling of $x, y$ by $L, \alpha_{q}$ and $\beta_{q}$ are independent of the size of the domain; they are given by $\Delta Q / Q_{0}$, where $\Delta Q$ is the change in water vapour along the zonal and the meridional direction for $\alpha_{q}$ and $\beta_{q}$ respectively. A domain with a high value of $Q_{0}$ (close to the tropics, for example) will have smaller values of $\alpha_{q}, \beta_{q}$. Scaling $q_{s}$ by the same factor as $\tilde{q}$, nondimensionalising and substituting into (9), we get

$$
\begin{array}{r}
\frac{D}{D t}\left(\nabla^{2} \eta-\eta\right)+\eta_{x}-\delta \tilde{q}=0, \\
\frac{D \tilde{q}}{D t}=\beta_{q} v+\alpha_{q} u-\delta \tilde{q} .
\end{array}
$$

These equations form the basis of our analysis in the following sections.

\section{Linear Analysis}

Linearising (10) about a saturated state of rest, we obtain

$$
\begin{array}{r}
\frac{\partial}{\partial t}\left(\nabla^{2}-1\right) \eta+\eta_{x}=\delta \tilde{q} \\
\frac{\partial \tilde{q}}{\partial t}-\beta_{q} \eta_{x}-\alpha_{q}\left(-\eta_{y}\right)=-\delta \tilde{q}
\end{array}
$$

where the geostrophic velocity has been expressed in terms of $\eta$. In the non-condensing phase, i.e., with $\delta=0$, the equations in (11) are uncoupled and reduce to the passive advection of moisture by Rossby waves. With $\delta=1$, the condensing phase, on Fourier transforming in space and time and re-arranging terms yields

$$
\left[\begin{array}{cc}
\frac{-k_{x}}{1+k_{x}^{2}+k_{y}^{2}} & \frac{-i}{1+k_{x}^{2}+k_{y}^{2}} \\
-k_{x} \beta_{q}+k_{y} \alpha_{q} & -i
\end{array}\right]\left[\begin{array}{l}
\hat{\eta} \\
\hat{\tilde{q}}
\end{array}\right]=\sigma\left[\begin{array}{l}
\hat{\eta} \\
\hat{\tilde{q}}
\end{array}\right]
$$

Where $k_{x}, k_{y}$ are the spatial wavenumbers and $\sigma$ is the phase speed of the wave. Fourier transformed quantities are represented with a hat. In the absence of gradients, the matrix on the left hand side is upper triangular, and the eigenvalues are given by the diagonal elements. Thus, the eigenvalues are $\left\{-k_{x} /\left(1+k_{x}^{2}+k_{y}^{2}\right),-i\right\}$, which correspond to a dry Rossby wave and an exponentially damped mode, respectively. The eigenvector corresponding to the Rossby wave is of the form $e_{R}=\left[\begin{array}{ll}c & 0\end{array}\right]^{T}$ for arbitrary real-valued $c$, indicating a decoupling of the Rossby wave from the effects of condensation. Thus, this analysis immediately points to the crucial role of moisture gradients in the present non-divergent framework.

\section{Purely meridional gradients $\left(\alpha_{q}=0, \beta_{q} \neq 0\right)$}

We begin by considering only meridional gradients in the saturation profile, i.e., $\beta_{q} \neq 0, \alpha_{q}=0$. Taking the limit of large $k_{x}$ in (12), we see that in both rows of the matrix on the LHS, the term in the second column is small as compared the term in the first column, and hence the effects of condensation are minimal. Therefore, for strong coupling between moisture and $\eta$, much of our attention is restricted modes with small values of $k_{x}$, i.e, those with a large zonal scale.

The dispersion relation for two representative cases $\left(\beta_{q}=0.2,0.8\right)$ is shown in Figure 2. The differences between these moist waves and dry Rossby waves are noted below:

- One mode is very weakly damped (almost neutral), while the other is strongly damped (with $\mathcal{O}(1)$ damping). In particular, waves associated with the first eigenvector are strongly damped for $k_{x}<0$ and those associated with the second eigenvector are strongly damped for $k_{x}>0$ for $\beta_{q}<0.5$. This situation is reversed when $\beta_{q}>0.5$.

- The corresponding phase relationship between the meridional velocity $v$ and $\tilde{q}$ is shown in Figure 3 for $\beta_{q}=0.2,0.8$ respectively. For the strongly damped waves, the meridional velocity and condensation are in quadrature. Thus, the condensation occurs closer to the centre of the Rossby gyre rather than the flanks, this appears to strongly damp the wave. For the almost neutral wave, the meridional velocity and condensation are nearly in phase. Thus, the condensation is co-incident with the meridional velocities at the flanks of the Rossby gyres which results in a marginal damping, but slows down the wave.

- Figure 3 also shows the dispersion relation (for a fixed $k_{y}$ ). Clearly, as $\beta_{q}$ increases, the frequency corresponding to the neutral modes decreases, i.e., the phase speed decreases with a stronger gradient [consistent with 19]. Further, as reported, $v$ and $\tilde{q}$ are almost perfectly in phase. Note that we are referring to the phase difference for small $k_{x}$, and not the asymptotic value as $k_{x}$ becomes large. In 
fact, as $\beta_{q} \rightarrow 1$, the absolute mimimum phase difference (around $\left|k_{x}\right| \approx 2$ ) tends to zero (Figure 4). This is also the case when $k_{y}$ is increased keeping $\beta_{q}$ constant (not shown).

- As $\beta_{q}$ decreases, the absolute mimimum phase difference between $v$ and $\tilde{q}$ increases from zero, and reaches a minimum value between $\pi / 4$ and $\pi / 2$ for the fastest modes as shown in Figure 4. Concomitantly, the frequency of the moist waves approaches that of dry Rossby waves.

The decrease in phase speed with increasing $\beta_{q}$ can be qualitatively understood by examining (11). Specifically, since $v$ and $\tilde{q}$ have the same phase as $\beta_{q} \rightarrow 1$ the tendency term in the PV equation becomes small and results in a slowly or almost non-propagating wave. More clearly, the reduction in phase speed of the moist modes in the presence of condensation can also be understood by linearising the potential vorticity (PV) of the full moist shallow water equations. For a state of rest, the moist $\mathrm{PV}$ is given by:

$$
\begin{aligned}
\mathrm{PV} & =\frac{f_{0}+\beta y}{H-\chi Q_{0}\left(1-\beta_{q} y\right)}, \\
& =\frac{f_{0}+\beta y}{H_{q}\left(1+M_{\beta} y\right)}, \\
& \approx\left(f_{0}+\beta y\right)\left(1-M_{\beta} y\right) / H_{q}, \\
& \approx\left(f_{0}+\left(\beta-f_{0} M_{\beta}\right) y\right) / H_{q},
\end{aligned}
$$

where $H_{q}=H_{0}-\chi Q_{0}$ and $M_{\beta}=\beta_{q} \chi Q_{0} / H_{q}$. Thus, we see that the moisture gradient counters the effect of the dynamical $\beta$, similar to the effect of a sloping bottom topography. This implies that the advection of planetary vorticity by the winds generated by the propagation of the Rossby wave is counteracted by condensation/drying occuring due to the same winds. Therefore, there is a smaller net increase/decrease in PV, resulting in slower Rossby wave propagation. We note that if $\beta_{q}>1$, the planetary vorticity advection is overwhelmed by the condensation, and an eastward moving unstable mode results. However, in our system we require $\beta_{q} \leq 1$, and therefore this situation does not occur.

\section{Purely zonal gradients $\left(\alpha_{q} \neq 0, \beta_{q}=0\right)$}

The dispersion relation for $\alpha_{q}=0.1,0.9$ are shown in Figure 5. Note that our notation is such that a positive $\alpha_{q}$ corresponds to a decrease in the saturation vapour to the east and vice versa. The differences from the previous case with only a meridional gradient are noted below:

- The inclusion of a zonal gradient results in an instability (marked by the filled contours in Figure 5), and the area in the wavenumber space covered by
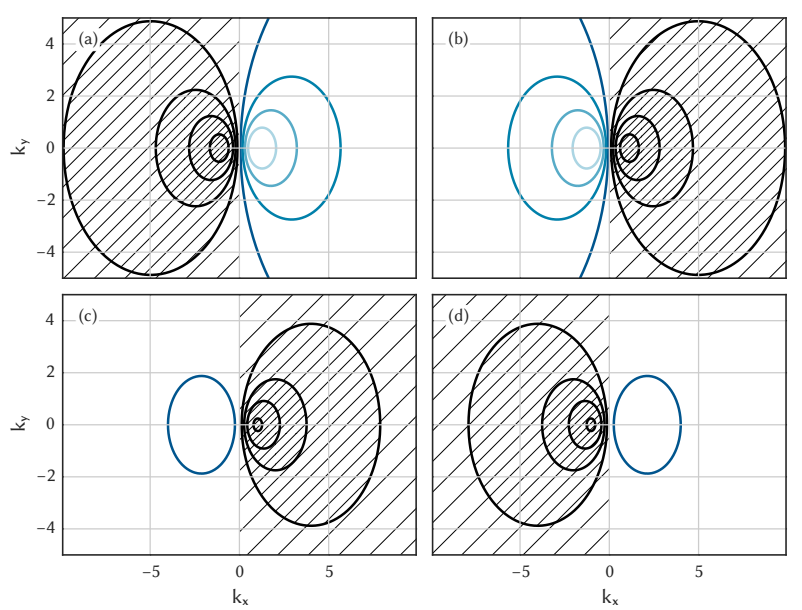

FIG. 2: Phase speed $(\Re(\sigma))$ corresponding to $\alpha_{q}=0$ and $\beta_{q}=0.2$ is plotted in panels $\mathbf{a}, \mathbf{b}$ and $\beta_{q}=0.8$ in panels $\mathbf{c}, \mathbf{d}$. The left panels correspond to eigenvector 1 and the right panels correspond to eigenvector 2 . The region of the $k_{x}-k_{y}$ plane where the modes are strongly damped $(\Im(\sigma)<-0.1)$ is hatched.
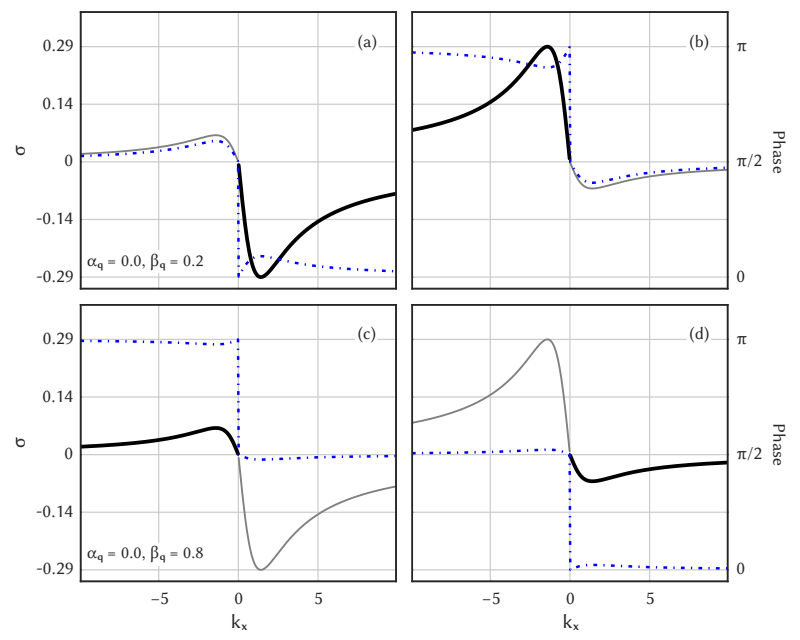

FIG. 3: Phase speed $(\Re(\sigma))$ and phase relationship between $v$ and $\tilde{q}$ for $k_{y}=2$. Panels $\mathbf{a}, \mathbf{b}$ correspond to $\alpha_{q}, \beta_{q}=0.0,0.2$ and $\mathbf{c}, \mathbf{d}$ correspond to $\alpha_{q}, \beta_{q}=0.0,0.8$. $\Re(\sigma)$ is plotted in thick black and thin gray lines for the almost neutral and strongly damped modes respectively. The phase difference is plotted in blue dash-dot lines.

the instability increases with increasing $\alpha_{q}$. Further, inspecting Figure 5, we note that for small $\alpha_{q}$ the unstable modes propagate westward, while for large $\alpha_{q}$ there emerges an eastward propagating unstable mode with a large zonal scale.

- The phase relation in Figure 6a,c suggests that both westward and eastward unstable modes are characterised by $v$ leading $\tilde{q}$ by a value greater than $\pi$. This is in contrast to a lag in the previous case (with $\alpha_{q}=0, \beta_{q} \neq 0$ ). 


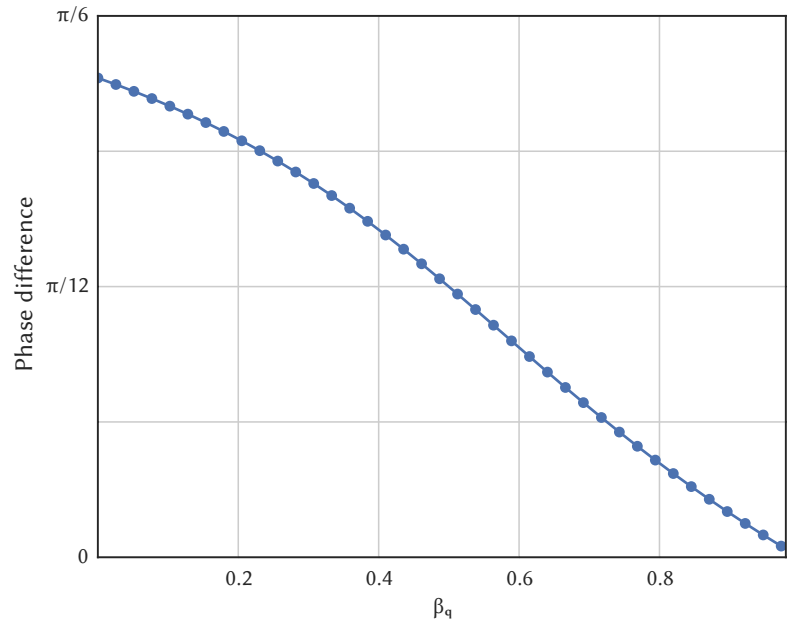

FIG. 4: The variation of the maximum of the phase difference between $v$ and $\tilde{q}$ with $\beta_{q}$.

- Examining Figure 6, we note that the dispersion relation of the unstable mode resembles that of a dry Rossby wave, but is shifted along the $k_{x}$-axis. This shift is more pronounced as $\alpha_{q}$ increases (seen by comparing panels a and $\mathbf{c}$ of Figure 6).

- When the sign of $\alpha_{q}$ is reversed, the dispersion relation is reflected about the line $k_{x}=0$ (not shown). Therefore, the direction of propagation in the zonal direction remains unchanged and is reversed in the meridional direction.

At a phase lag greater than $\pi$, the tendency terms are such that a positive (negative) value of $\mathrm{PV}$ is associated with a positive (negative) tendency, and therefore the PV field grows with time. The reversal in the meridional direction of propagation on changing the sign of $\alpha_{q}$ can be explained by noting that the zonal geostrophic winds are maximum on the northward and southward flanks of a Rossby ridge/trough.

\section{Zonal and meridional gradients $\left(\alpha_{q}, \beta_{q} \neq 0\right)$}

In a situation where both gradients are present, the behaviour is a mixture of the two previously considered cases. As seen in Figure 7, if $\beta_{q}$ is increased while keeping $\alpha_{q}$ constant, the range of wavenumbers for which the unstable waves are eastward increases, while the overall range of wavenumbers that are unstable is reduced. Further, there is a change in the direction of propagation of the most unstable modes with both $\alpha_{q}$ and $\beta_{q}$. Specifically, holding $\alpha_{q}$ fixed, the most unstable mode lies in the second and fourth quadrants of Figure $7 \mathbf{a}, \mathbf{b}$ and in the first and third quadrants of Figure $7 \mathbf{c}, \mathbf{d}$. Thus, for $\beta_{q}=0.2\left(\beta_{q}=0.6\right)$ the most unstable mode is eastward (westward), with the change occuring at $\beta_{q}=0.5$.
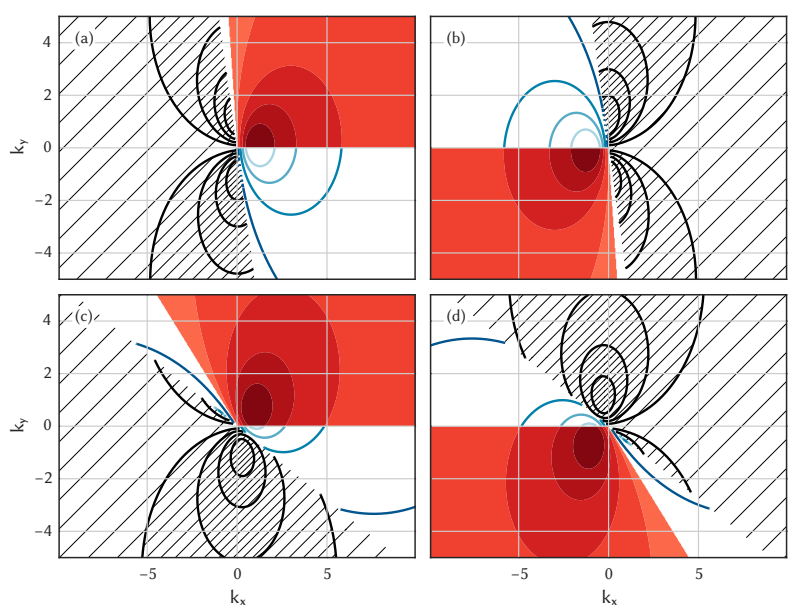

FIG. 5: Phase speed $(\Re(\sigma))$ corresponding to $\beta_{q}=0$ and $\alpha_{q}=0.1$ is plotted in panels $\mathbf{a}, \mathbf{b}$ and $\alpha_{q}=0.9$ in panels $\mathbf{c}, \mathbf{d}$. The left panels correspond to eigenvector 1 and the right panels correspond to eigenvector 2 . The region of the $k_{x}-k_{y}$ plane where the modes are strongly damped $(\Im(\sigma)<-0.1)$ is hatched. The region of the $k_{x}-k_{y}$ plane where the modes are unstable $(\Im(\sigma)>0)$ is shaded red.
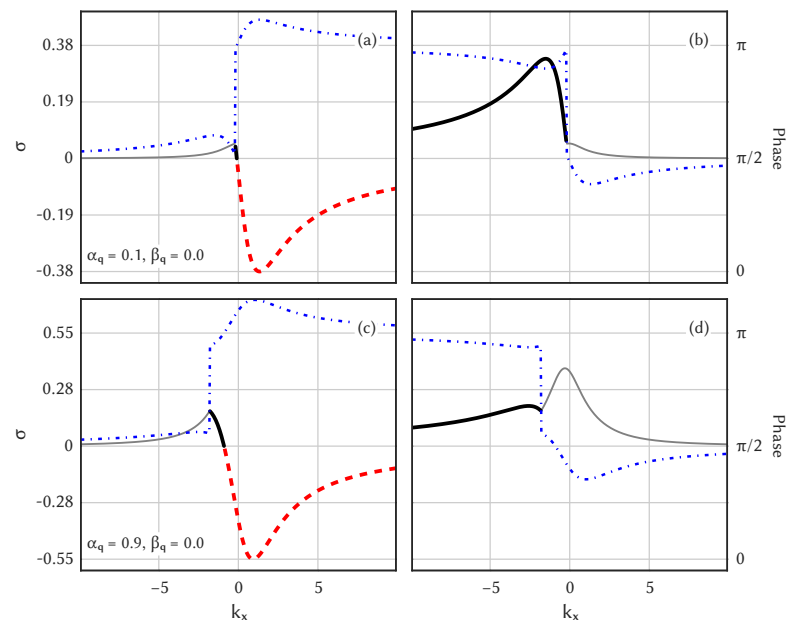

FIG. 6: Phase speed $(\Re(\sigma))$ and phase relationship between $v$ and $\tilde{q}$ for $k_{y}=2$. Panels a,b correspond to $\alpha_{q}, \beta_{q}=0.1,0.0$ and $\mathbf{c}, \mathbf{d}$ correspond to $\alpha_{q}, \beta_{q}=0.9,0.0$. Mildly damped, strongly damped and unstable modes are plotted in bold black, thin grey and dashed red lines respectively. The phase difference is plotted in blue dash-dot lines.

Similarly, holding $\beta_{q}$ fixed, the direction of propagation reverses with a change in sign of $\alpha_{q}$ (not shown). Quantitatively, the maximum non-dimensional growth rate is $\approx 0.04$ for $\left(\alpha_{q}, \beta_{q}=0.4,0.2\right)$ and $\approx 0.035$ for $\left(\alpha_{q}, \beta_{q}=0.4,0.6\right)$, which, with an advective timescale of the order of a day or two, implies these modes mature in 25-50 days. Further, in both cases, these are very large scale modes whose dimensional phase speed is $\approx 1-3$ $\mathrm{m} / \mathrm{s}$.

As seen in Figure 1, and from observations in the trop- 

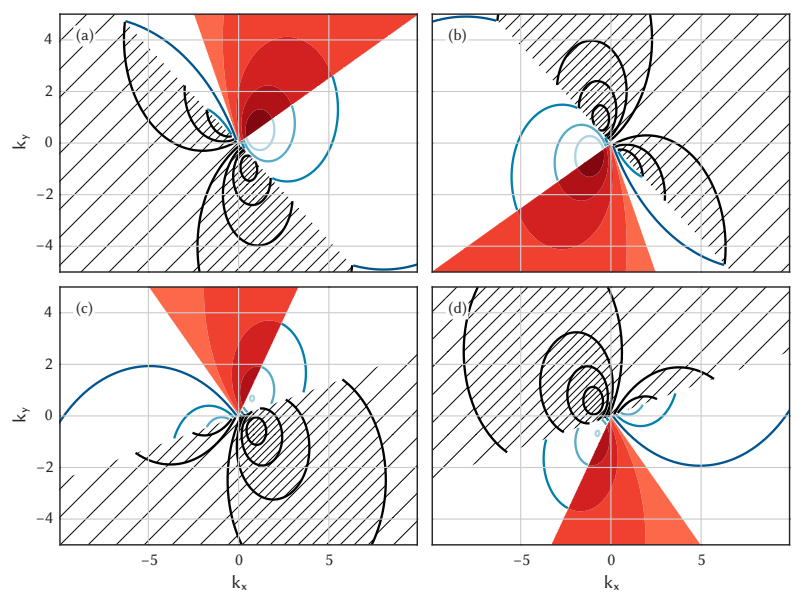

FIG. 7: $\quad$ Phase speed $(\Re(\sigma))$ corresponding to $\alpha_{q}, \beta_{q}=0.4,0.2$ is plotted in panels $\mathbf{a}, \mathbf{b}$ and $\alpha_{q}, \beta_{q}=0.4,0.6$ in panels $\mathbf{c}, \mathbf{d}$. The left panels correspond to eigenvector 1 and the right panels correspond to eigenvector 2. The region of the $k_{x}-k_{y}$ plane where the modes are strongly damped $(\Im(\sigma)<-0.1)$ is hatched. The region of the $k_{x}-k_{y}$ plane where the modes are unstable $(\Im(\sigma)>0)$ is shaded red.
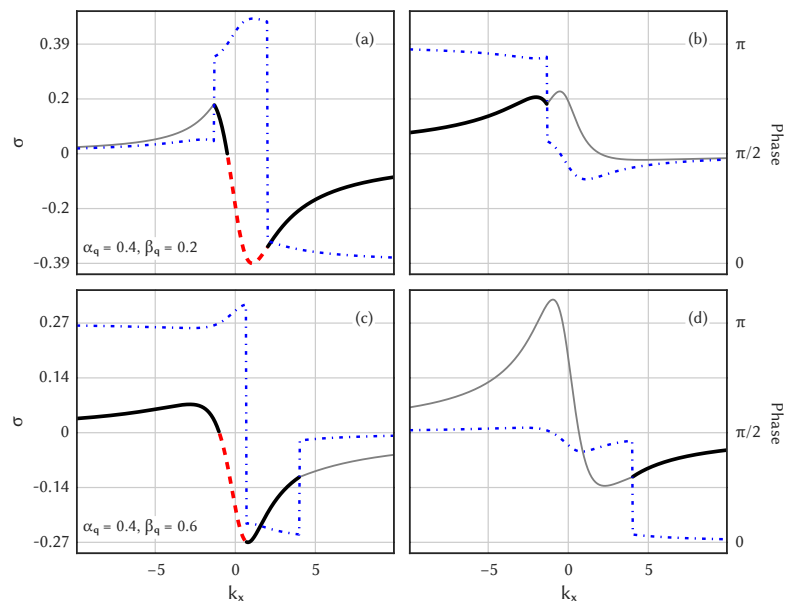

FIG. 8: Phase speed $(\Re(\sigma))$ and phase relationship between $v$ and $\tilde{q}$ for $k_{y}=2$. Panels $\mathbf{a}, \mathbf{b}$ correspond to $\alpha_{q}, \beta_{q}=0.4,0.2$ and $\mathbf{c}, \mathbf{d}$ correspond to $\alpha_{q}, \beta_{q}=0.4,0.6$. Mildly damped, strongly damped and unstable modes are plotted in bold black, thin grey and dashed red lines respectively. The phase difference is plotted in blue dash-dot lines.

ics [18], the atmosphere is characterised by small values of $\alpha_{q}$ and relatively larger values of $\beta_{q}$, with $\beta_{q}$ ranging between 0.5 and 0.7 and $\alpha_{q}$ ranging between 0.3 and 0.4 . From the above discussion on the direction of propagation of the most unstable mode, noting that $\beta_{q}>0.5$, this implies that in regions with a negative (positive) $\alpha_{q}$, i.e, moisture increasing (decreasing) towards the east, the most unstable modes are eastward (westward). Thus, as was noted in a tropical setting [19], the modes grow in the direction of increasing moisture.

\section{The mechanism of the instability}

In our nondivergent system, there are three terms that play a role in the balance of PV. Namely, the advection of planetary vorticity, condensation in the meridional direction and condensation in the zonal direction. This is made clear by looking at the linearised PV with both gradients present:

$$
\begin{aligned}
\mathrm{PV} & =\frac{f_{0}+\beta y}{H-\chi Q_{0}\left(1-\beta_{q} y-\alpha_{q} x\right)}, \\
& =\frac{f_{0}+\beta y}{H_{q}\left(1+M_{\beta} y+M_{\alpha} x\right)}, \\
& \approx\left(f_{0}+\beta y\right)\left(1-M_{\beta} y-M_{\alpha} x\right) / H_{q}, \\
& \approx\left(f_{0}+\left(\beta-f_{0} M_{\beta}\right) y-f_{0} M_{\alpha} x\right) / H_{q},
\end{aligned}
$$

where $H_{q}=H_{0}-\chi Q_{0}, M_{\alpha}=\alpha_{q} \chi Q_{0} / H_{q}$ and $M_{\beta}=$ $\beta_{q} \chi Q_{0} / H_{q}$.

In the meridional direction, condensation and vorticity advection have opposing tendencies, and thus a larger $\beta_{q}$ leads to a reduction in phase speed. By definition, $\beta_{q} \leq 1$ and so we do not experience an instability. In the zonal direction, however, there is no compensating term to balance condensation. Hence, the introduction of a nonzero gradient in the zonal direction immediately results in the generation of unstable modes. Similarly, with gradients in both directions, the instability arises at certain combinations of the moisture gradients where the contribution to the $\mathrm{PV}$ field by the dynamical $\beta$ is smaller than the contribution by the condensation.

\section{SUMMARY AND DISCUSSION}

In this article, we have presented the derivation of a forced QG system that highlights constraints placed on the forcing function that are required to maintain geostrophic balance at leading order. The forcing is a due to a dynamically active scalar which mimics the action of condensation, thus providing a simple model for the moist atmosphere. We emphasize that the constraints derived here - in particular, the slow nature of the timescale of condensation - have been used in previous studies of forced QG and shallow water turbulence [31], but normally without a formal justification.

Given that the time scale of condensation turns out to be comparable to that of advection, moisture anomalies are not immediately relaxed to zero, as is the case with models that enforce SQE. Further, in the present model, heating is completely driven by non-divergent advection. In the presence of purely meridional moisture gradients, we only obtain damped and neutral modes. The neutral moist waves are noted to be slower than dry Rossby waves due to a reduction in an "equivalent gradient" of PV. This is in contrast to SQE-based systems where a reduction in the equivalent depth is the cause for slower 
convectively coupled waves [2]. Further, the addition of meridional gradients on a $\beta$-plane complements the work of [5] by showing that the unstable modes on a $f$-plane are stabilised in the presence of a planetary vorticity gradient.

This idea of "diabatic PV gradients" has been previously explored in $[14,15]$ while studying the effects of latent heat release on baroclinic wave development. In both these cases, Rossby waves are generated purely due to diabatic processes and propagate via the advection of moisture and the resultant heating by wind anomalies. This, is in some sense analogous to the results of [5]. The inclusion of boundaries moderates the instability, as suggested in [14] and proved mathematically in [27]. This is due to the influence of the Rossby edge wave, which provides an opposing/stabilising influence. These studies provide an intriguing parallel to our own in terms of the presence of stabilising/destabilising PV gradients, though it is unclear whether they can be reduced to a similar mathematical structure.

The inclusion of gradients in the zonal direction, by themselves or in combination with meridional gradients, is seen to result in the formation of unstable modes. The nature of the instability is deduced from the PV equation; specifically, an unstable situation occurs when the contribution to PV from condensation overwhelms the stabilising dynamical $\beta$ contribution. It is seen that both zonal and meridional gradients affect the direction of propagation of the most unstable mode, In particular, for gradients that roughly correspond to atmospheric observations, the most unstable mode propagates zonally in the direction of increasing moisture. Further, the most unstable mode has a large spatial scale, small phase speed (of the order of a few $\mathrm{m} / \mathrm{s}$ ) and matures over intraseasonal timescales.

The limitations of the study pertain mainly to the geometry of the domain under consideration and the use of a linear gradient in $Q_{s}$ which enables us to obtain analytical results. To facilitate analysis in the Fourier domain, we use $\delta=1$ throughout, which is not representative of geophysical situations where precipitating and non-precipitating regions often co-exist. However, we note that the mechanism in terms of the PV budget of the waves seems robust enough to apply in more general situations. Indeed, slowdown of Rossby waves is seen in the eigenanalysis on an equatorial $\beta$ with meridional moisture gradients [19], and preliminary numerical work on the QG system shows a slowdown of the Rossby waves even in the presence of non-precipitating regions. A study using spherical geometry with more realistic gradients would also be interesting from a physical perspective. Similarly, the analysis of the two layer case with baroclinic instability and the interaction of Rossby waves which advect both PV and moisture appear to be fruitful avenues of study.

Acknowledgements: The authors thank the two reviewers and the editor for comments which greatly added to the content and presentation of this work, especially in relating our work to extant work on diabatic Rossby waves. We thank Raghu Murtugudde for helpful discussions. We thank Dargan Frierson whose suggestions helped guide the approach and presentation of the paper. We thank Mike Wallace for discussions that helped us gain physical insight into the problem, enabling us to sketch out the underlying mechanisms.
[1] A. E. Gill, Geophysical \& Astrophysical Fluid Dynamics 19, 119 (1982), ISSN 0309-1929.

[2] G. N. Kiladis et al., Reviews of Geophysics 47 (2009), ISSN 8755-1209.

[3] A. J. Majda and S. N. Stechmann, in Intraseasonal Variability in the Atmosphere-Ocean Climate System, edited by W. K. M. Lau and D. E. Waliser (Springer, 2012), pp. 549-568.

[4] J. D. Neelin and N. Zeng, Journal of the atmospheric sciences 57, $1741(2000)$.

[5] A. H. Sobel, J. Nilsson, and L. M. Polvani, Journal of the atmospheric sciences 58, 3650 (2001).

[6] D. J. Raymond and . Fuchs, Tellus A 59, 627 (2007), ISSN 1600-0870.

[7] P. R. Bannon, Journal of the Atmospheric Sciences 43, 2261 (1986), ISSN 0022-4928.

[8] J. Lambaerts, G. Lapeyre, and V. Zeitlin, Journal of the Atmospheric Sciences 68, 1234 (2011), ISSN 0022-4928.

[9] M. Fantini, Journal of the Atmospheric Sciences 50, 1199 (1993), ISSN 0022-4928.

[10] J. Lambaerts, G. Lapeyre, and V. Zeitlin, Journal of the Atmospheric Sciences 69, 1405 (2012), ISSN 0022-4928.

[11] W. J. Gutowski, L. E. Branscome, and D. Stewart, Journal of the Atmospheric Sciences 49, 306 (1992), ISSN 0022-4928.

[12] G. Lapeyre and I. Held, Journal of the Atmospheric Sciences
61, 1693 (2004), ISSN 0022-4928.

[13] G. Craig and H.-R. Cho, Journal of the Atmospheric Sciences 45, 2622 (1988).

[14] C. Snyder and R. S. Lindzen, Journal of the Atmospheric Sciences 48, 76 (1991), ISSN 0022-4928.

[15] D. J. Parker and A. J. Thorpe, Journal of the Atmospheric Sciences 52, 1699 (1995).

[16] J. S. Sabato, Journal of the Atmospheric Sciences 65, 1378 (2008), ISSN 0022-4928, 1520-0469.

[17] J. L. Mitchell et al., Nature Geoscience 4, 589 (2011).

[18] J. Sukhatme, Quarterly Journal of the Royal Meteorological Society 139, 414 (2012).

[19] J. Sukhatme, Quarterly Journal of the Royal Meteorological Society 140, 1838 (2013), ISSN 1477-870X.

[20] F. Bouchut, J. Lambaerts, G. Lapeyre, and V. Zeitlin, Physics of Fluids 21, 116604 (2009), ISSN 10706631.

[21] A. E. Gill et al., Geophysical \& Astrophysical Fluid Dynamics 12, 195 (1979), ISSN 0309-1929.

[22] C. J. Muller et al., Geophysical Research Letters 36, L16804 (2009), ISSN 1944-8007.

[23] C. Holloway and D. Neelin, Journal of the Atmospheric Sciences 67, 1091 (2009).

[24] D. M. W. Frierson, A. J. Majda., and O. M. Pauluis, Communications in Mathematical Sciences 2, 591 (2004). 
[25] J. Sukhatme and W. R. Young, Quarterly Journal of the Royal Meteorological Society 137, 1561 (2011), ISSN 1477-870X.

[26] K. A. Emanuel, J. D. Neelin, and C. S. Bretherton, Quarterly Journal of the Royal Meteorological Society 120, 1111 (1994), ISSN 1477-870X.

[27] H. De Vries, J. Methven, T. H. A. Frame, and B. J. Hoskins, Journal of the Atmospheric Sciences 67, 2766 (2010).

[28] J.-I. Yano, S. Mulet, and M. Bonazzola, Tellus A 61, 417 (2009), ISSN 02806495, 16000870.

[29] B. Mapes et al., Dynamics of Atmospheres and Oceans 42, 3 (2006), ISSN 03770265.

[30] R. S. Lindzen, Journal of the atmospheric sciences 60, 3009 (2003).

[31] A. P. Showman, Journal of the Atmospheric Sciences 64, 3132
(2007), ISSN 0022-4928, 1520-0469.

[32] R. K. Scott and L. M. Polvani, Journal of the Atmospheric Sciences 64, 3158 (2007).

[33] E. D. Maloney, Journal of Climate 22, 711 (2009).

[34] J. A. Andersen and Z. Kuang, Journal of Climate 25, 2782 (2011).

[35] M. S. Pritchard and C. S. Bretherton, Journal of the Atmospheric Sciences 71, 800 (2014).

[36] A. Sobel, S. Wang, and D. Kim, Journal of the Atmospheric Sciences 71, 4276 (2014).

[37] L. C. Hirons, P. Inness, F. Vitart, and P. Bechtold, Quarterly Journal of the Royal Meteorological Society 139, 1417 (2013). 